\title{
Inhaled disodium cromoglycate as maintenance therapy for childhood asthma: time to consign to history?
}

Disodium cromoglycate was introduced in the late 1960s as maintenance therapy for persistent asthma and as a modifier of exercise induced bronchoconstriction. It was the first treatment designed to manage the chronic nature of the disease and modify the inflammatory component without untoward systemic side effects. After its antiinflammatory effects had been established, ${ }^{1}$ clinical trial data began to appear in the late 1960s and, although it remains as an option in the 1995 British guidelines on asthma management, ${ }^{2}$ its use was already in steep decline ${ }^{3-4}$ and it has now become a minority therapeutic option. In the current issue of Thorax a systematic review is presented which argues against its continuation as an option for the management of persistent symptoms in childhood. ${ }^{5}$ Why has it taken so long for the evidence of few, if any, beneficial effects in the management of persistent childhood asthma to appear in the face of its virtual disappearance from the prescribing armamentarium? What lessons can be drawn from the now passing cromoglycate era?

In the years immediately after introduction to the market of any new therapeutic agent and when interest is intense it is likely that the evidence presented to the prescribing community is selective. Indeed, the present review provides evidence for this as the older studies were more likely to show a beneficial effect than those performed in recent years. ${ }^{5}$ The paucity of data in very young children in the early years of the cromoglycate era is not unique to this single therapeutic agent. A large amount of "off label" and unlicensed prescribing is commonplace in childhood and this is more acute in the younger child population where clinical trial data are sparse. ${ }^{6}$ This can clearly be seen in the present review as studies in younger children only began to appear in more recent years when studies designed by practising clinicians with or without industry support sought to correct this deficiency. For asthma and wheezing illness the paucity of clinical trial data is particularly frustrating as most childhood asthma presents in the first four years of life. Consequently, it is in this youngest age group that extrapolation from trial data in older children and adults is required, which clearly presents a problem for practitioners wishing to engage in evidence based prescribing.

Although meta-analyses such as that reported in the current issue ${ }^{5}$ are essential, practitioners managing childhood asthma must also pay close attention to both the quality of the studies included in such an analysis and also whether the patients included in the published trials describe the kind of patients typically seen in their surgery or clinic. As most recruitment to clinical trials is based in hospital clinics while the majority of patients are managed in primary care, this poses a problem in generalising results from what are likely to be the relatively small proportion of more severely affected individuals to the majority with mild to moderate disease. Inclusion of less severely affected individuals from primary care in order to assess established therapeutic approaches has resulted in rather different outcomes from those found in more severely affected hospital based populations and from studies in adult populations. ${ }^{78}$

Studies required for licensing purposes are required to demonstrate efficacy by comparing "hard" outcomes such as peak expiratory flow rate or forced expiratory volume in one second against placebo. Such studies are tightly regu- lated and are usually associated with a significant benefit for placebo, a likely consequence of improved compliance with treatment during the close monitoring required during such trials. Such tightly regulated conditions are far removed from the real world where compliance with treatment is generally poor, even when entirely supervised by parents. ${ }^{9}$ Added to this problem are patient/parent preferences and selective administration. Although the side effects of cromoglycate appear to be minor, it is likely that they have a significant impact on compliance with treatment. These side effects include an unpleasant bitter taste and upper respiratory tract irritation including cough soon after administration. ${ }^{5}$ In combination with the 3-4 times a day regime required for maximum benefit, it is no wonder that this treatment has become unpopular with children, parents, and prescribers.

The evidence for the efficacy and overall effectiveness (efficacy $\times$ compliance) of cromoglycate in persistent childhood asthma is weak. However, its role in exercise induced bronchoconstriction has not been addressed in the current review and it is likely that this will remain an option for this particular feature of asthma. Rather than waiting for more than 30 years for evidence of effectiveness in the youngest age group of children which make up so much of the work load in primary ${ }^{10}$ and secondary care, ${ }^{11}$ further studies on the effectiveness of more recently introduced formulations such as long acting $\beta$ agonists and leukotriene receptor antagonists need to be performed, particularly in young children, in order to inform evidence based guidelines and support good prescribing practice.

Recent changes in legislation on drug licensing in the United States ${ }^{12}$ and recommendations from the Committee for Proprietary Medical Products (CPMP) of the European Union $^{13}$ should provide a stimulus for further studies in children of all ages and particularly in the youngest age groups where data are particularly sparse.

PETER J HELMS

Department of Child Health,

University of Aberdeen,

Foresterhill, Aberdeen AB25 2ZD, UK

1 Cox JSG. Disodium cromoglycate (FPL 670) ('Intal'): a specific inhibition of reaginic antibody-antigen mechanisms. Nature 1967;216:1328-9.

2 British Thoracic Society, National Asthma Campaign, Royal College of Physicians of London, et al. The British guidelines on asthma management: Physicians of London, et al. The British guidelines on asthma managem
1995 review and position statement. Thorax 1997;52(Suppl 1):S1-21. 1995 review and position statement. Thorax 1997;52(Suppl 1):S1-21.
3 Warner JO. Review of prescribed treatment for children with asthma in 1990. BMF 1995;311:633-6.

4 Kaarsgaren RJ, Zulstra RF, Helms P. Asthma medication in children: 1991. Respir Med 1994;88:383-6.

5 Tasche MJA, Uijen JHJM, Bernsen RMD, et al. Inhaled sodium cromoglycate as maintenance therapy in children with asthma. Thorax 2000;55:913-20.

Sutcliffe AG Prescribing medicines for children. BMF 1999:319:70-1.

7 Doull IJM, Lampe FC, Smith S, et al. Effect of inhaled corticosteroids on episodes of wheezing associated with viral infection in school age children: randomised double blind placebo controlled trial. BMf 1997;315:858-62.

8 Verberne AAPH, Frost C, Duiverman EJ, et al. Addition of salmeterol verVerberne AAPH, Frost C, Duiverman EJ, et al. Addition of salmeterol ver-
sus doubling the dose of beclomethasone in children with asthma. Am $\mathcal{f}$ sus doubling the dose of beclomethas
Respir Crit Care Med 1998;158:213-9.

Respir Crit Care Med 1998;158:213-9.
9 Gibson NA, Ferguson AA, Aitchison TC, et al. Compliance with inhaled asthma medication in preschool children. Thorax 1995;50:1274-9.

10 Hansell A, Hollowell J, Nichols T, et al. Use of the General Practice Research Database for respiratory epidemiology: a comparison with the 4th Morbidity Survey in General Practice. Thorax 1999;54:413-9.

11 Strachan DP, Anderson HR. Trends in hospital admission rates for asthma in children. BMF 1992;304:819-20.

12 Kauffman R. Status of drug approval processes and regulation of medications for children. Curr Opin Pediatr 1995;7:195-7.

13 European Medicines Evaluations Agency (EMEA). Note for guidance i6. Clinical investigation of medicinal products in children. London: EMEA, 1997. 